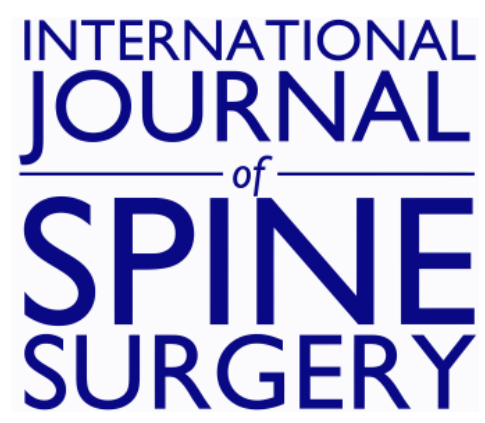

\title{
Osteomyelitis-Discitis at the Thoracolumbar Junction and the Development of Postinfectious Spinal Deformity: A Surgical Case Series
}

TERENCE VERLA, ROBERT NORTH, VENITA SIMPSON and ALEXANDER E. ROPPER

Int J Spine Surg 2020, 14 (4) 552-558

doi: https://doi.org/10.14444/7073

http://ijssurgery.com/content/14/4/552

This information is current as of April 26, 2023.

Email Alerts Receive free email-alerts when new articles cite this article. Sign up at:

http://ijssurgery.com/alerts

The International Journal of Spine Surgery

2397 Waterbury Circle, Suite 1,

Aurora, IL 60504, Phone: +1-630-375-1432 


\title{
Osteomyelitis-Discitis at the Thoracolumbar Junction and the Development of Postinfectious Spinal Deformity: A Surgical Case Series
}

\author{
TERENCE VERLA, MD, ROBERT NORTH, MD, PHD, VENITA SIMPSON, MD, ALEXANDER E. \\ ROPPER, MD \\ Department of Neurosurgery, Baylor College of Medicine, Houston, Texas
}

\begin{abstract}
Background: Progressive spinal deformity and neural compromise are the main indications for surgical management of vertebral osteomyelitis-discitis. However, when such pathology presents at the thoracolumbar (TL) junction, it remains unclear what the appropriate intervention is. The therapeutic dilemmas of decompression with or without instrumented fusion, the need for circumferential decompression and reconstruction, as well as the prognostic factors for progression of kyphosis, all remained ill-defined in the literature. The objective of this study is to evaluate risk factors for instrumentation at TL junction in spinal osteomyelitis-discitis.

Methods: A review of patients at a single center with osteomyelitis-discitis at the TL junction between 2014 and 2018 was performed. Patients were 18 years or older with infectious pathologies at T10 to L2.

Results: Sixteen patients were included. Indication for instrumentation included progression of kyphosis following prior laminectomy/medical management. Of the 16 patients, 4 patients received laminectomy at initial treatment versus 12 patients receiving medical management alone. All 4 patients receiving laminectomy experienced progressive kyphosis requiring revision with instrumented fusion versus only 4 of 12 of the medically managed. Laminectomy, epidural compression, and vertebral body collapse were significant risk factors for kyphosis progression requiring instrumentation. The average time to surgical intervention for the indication of progressive kyphosis was 2.6 months after prior laminectomy and 6 months after initiation of medical management.

Conclusions: Given the proclivity for kyphotic deformity at the TL junction, patients may benefit from long segment instrumentation in addition to decompression at the initial surgery. Laminectomy alone may hasten kyphosis progression.
\end{abstract}

Lumbar Spine

Keywords: osteomyelitis, thoraco-lumbar junction, spine fusion, spinal deformity

\section{INTRODUCTION}

Osteomyelitis-discitis continues to have devastating effects with increasing incidence owing to the ageing population and rising number of drug using and immunocompromised patients. ${ }^{1-3}$ With improvement in diagnostic tools, such as magnetic resonance imaging-guided and computed tomography-guided biopsy of affected spinal segments, in conjunction with advancement in antibiotic therapy, patient outcomes have historically improved. ${ }^{4-9}$ Upfront medical therapy, with antimicrobials, is the mainstay treatment for most patients. However, spinal instability and deformity, severe epidural compression, and neurologic decline have traditionally been used as indicators for immediate surgical management of vertebral osteomyelitis-discitis. ${ }^{3,10-14}$ Surgery is also appropriate in the subacute or chronic settings whereby there is recurrence of infection, failure of antibiotic therapy, or refractory pain. ${ }^{1-13}$ Controversy exists regarding the utility of surgical interventions, the surgical approach, the need for instrumentation, and the appropriate timing of intervention. Surgical management is used for restoration of spinal stability and expeditious improvement of neurologic function. ${ }^{15-22}$

The destructive nature of the affected disc and vertebral end plates by the infectious process is the key to the development of kyphosis and spinal instability. Preservation of the posterior tension band helps in the maintenance of overall stability. At the thoracolumbar (TL) junction, the lordosiskyphosis transition significantly increases the risk of 
segmental instability when there is injury to any column of the spine. Elsewhere in the spine, surgical treatment options for pyogenic spondylodiscitis have included posterior decompression alone, decompression plus fusion, ${ }^{17}$ and use of intervertebral graft material for fusion after extensive debridement of infected substrate. ${ }^{19,23-25}$ Given the unique geometry and biomechanics of the transitional TL junction, when such pathology occurs here, the appropriate intervention may be slightly different than the general treatment algorithms for spinal osteomyelitis-discitis. Should laminectomy only be performed even in the absence of deformity? Is short segmental instrumentation sufficient in the presence of deformity? Or should patients undergo a long construct instrumentation to prevent progression of kyphosis and the need for revision surgery? There is a paucity of data characterizing deformity progression following medical or surgical treatment of osteomyelitis-discitis at the TL junction. This study describes a series of cases of osteomyelitis-discitis at the thoracolumbar junction and the outcomes of medical care, decompression without and with fusion.

\section{PATIENTS AND METHODS}

This is a retrospective case series of consecutive patients with osteomyelitis-discitis at the TL junction between 2014 and 2018 at Baylor St. Luke's Medical Center (Houston, TX), with approval from the Institutional Review Board. Patient consent was not required because this was a retrospective study, without any patient identifying information. Patients were 18 years or older with pathologies from T10 to L2. The exclusion criteria included: history of previous surgery at the indicated level, patients lost to follow-up within a year after initial diagnosis, and absence of sufficient perioperative radiographs

Preoperative and intraoperative data for each patient were collected with the use of patient charts and computerized medical records. Baseline characteristics were documented for each patient, including age, sex, race, and body mass index. Pertinent operative details were also collected, including surgical level, procedure, and estimated blood loss. Clinical data and outcome measures, such as hospital length of stay, pathologic organisms involved, and duration of antibiotics, were analyzed. The Bilsky score was used to assess spinal epidural compression from either the abscess or bony/disc compression at the apex of the kyphosis or the most stenotic level. We recognize that this score is relevant to neoplastic compression, but it is a useful analog in lieu of a widely used score or matrix for infectious compression. Radiographic parameters, such as the segmental kyphosis, were measured for involved segments. The Cobb angle was measured (on supine radiographs) between the inferior end plate of the adjacent rostral normal vertebra and the superior end plate of the adjacent caudal normal vertebra. Surgery was indicated if the kyphosis progressed to greater than $10^{\circ}$ increase from baseline at diagnosis, destruction of vertebral end plates by more than $50 \%$, worsening stenosis at the apex of kyphosis, uncontrolled pain, and progressive neurologic deficits. A subgroup analysis was done to evaluate time to progression of kyphosis deformity in patients who had medical management (intravenous antibiotics) alone versus laminectomy with antibiotics at initial diagnosis.Parametric data were expressed as the mean \pm standard deviation, and were compared using the Student $t$ test. Nonparametric data are expressed as the median (interquartile range), and were compared using the Mann Whitney $U$ test. Nominal data were compared with the $\chi^{2}$ test. A $P$ value $<.05$ was considered statistically significant.

\section{RESULTS}

Sixteen patients met the inclusion criteria for the study. Seven patients were excluded from the study either because they were lost to follow-up or had incomplete radiographs. The average age was 51.7 years, with $19 \%$ being women. Severe back pain was the presenting symptom in most patients. A total of 6 patients had an initial infection at the L1 to L2 level, 5 patients at the T11 to T12 level, 4 patients at the T12 to L1 level, and 1 patient at the T10 to T11 level. All patients were immediately started on broad-spectrum intravenous antibiotics after blood and urine cultures were sent due to the suspicion for spinal infection from clinical and radiographic evidence. Of the patients with positive blood cultures, the most common isolated organism was methicillin-resistant Staphylococcus aureus (4 patients). Three patients had negative cultures, 2 of whom were previously on long-term antibiotics for nonspinal infections. Final diagnosis of osteomyelitis-discitis was made from the combination of neurologic presentation, radiographic features, and presence of an infection. Once culture organisms were identified, antibiotic was narrowed appropri- 
Table. Clinical data of 16 patients with osteomyelitis-discitis at the thoracolumbar junction.

\begin{tabular}{|c|c|c|c|c|c|c|c|c|c|}
\hline $\begin{array}{l}\text { Patient } \\
\text { No. }\end{array}$ & $\begin{array}{l}\text { Age, } \\
\text { y/Sex }\end{array}$ & $\begin{array}{l}\text { Infected } \\
\text { Segment }\end{array}$ & $\begin{array}{c}\text { Initial } \\
\text { Management }\end{array}$ & Approach & $\begin{array}{l}\text { Indication } \\
\text { for Surgery }\end{array}$ & Organisms & $\begin{array}{c}\text { EBL, } \\
\text { mL }\end{array}$ & $\begin{array}{l}\text { Hospital } \\
\text { LOS, } \\
\text { Days }\end{array}$ & $\begin{array}{c}\text { Duration } \\
\text { of } \mathbf{A b x} \text {, } \\
\mathbf{w k}\end{array}$ \\
\hline 1 & $66 / \mathrm{M}$ & L1-2 & L1-L2 laminectomy & Combined & Kyphosis progression & GBS & 1450 & 44 & 8 \\
\hline 2 & $26 / \mathrm{M}$ & L1-2 & T12-L4 laminectomy & Combined & Prevent kyphosis & $\begin{array}{l}\text { ESBL Escherichia } \\
\quad \text { coli }\end{array}$ & 1100 & 10 & 8 \\
\hline 3 & $57 / \mathrm{M}$ & T12-L1 & T12-L1 laminectomy & Combined & Kyphosis progression & Candida & 2600 & 19 & 6 \\
\hline 4 & $44 / \mathrm{M}$ & L1-2 & L1-L2 laminectomy & Combined & Kyphosis progression & MSSA & 650 & 18 & 6 \\
\hline 5 & $20 / \mathrm{F}$ & L1-2 & Antibiotic & Posterior & Kyphosis progression & MSSA, Pseudomonas & 1500 & 58 & 8 \\
\hline 6 & $71 / \mathrm{F}$ & T12, L2-L3 & Antibiotic & Combined & Kyphosis progression & Pseudomonas & 1500 & 29 & $\begin{array}{l}2 \text { (completed } \\
\text { prior Abx) }\end{array}$ \\
\hline 7 & $60 / \mathrm{M}$ & $\mathrm{T} 12-\mathrm{L} 1$ & Antibiotic & Combined & Kyphosis progression & $\begin{array}{l}\text { Negative, prior } \\
\text { ESBL } E \text { coli }\end{array}$ & 1400 & 10 & 8 \\
\hline 8 & $54 / \mathrm{F}$ & $\mathrm{T} 11-\mathrm{T} 12$ & Antibiotic & Combined & Kyphosis progression & MRSA & 1000 & 35 & 8 \\
\hline 9 & $43 / \mathrm{M}$ & $\mathrm{T} 10$ & Antibiotic & Medical & $\mathrm{N} / \mathrm{A}$ & TB & $\mathrm{N} / \mathrm{A}$ & $\mathrm{N} / \mathrm{A}$ & 6 \\
\hline 10 & $53 / \mathrm{M}$ & $\mathrm{T} 11-\mathrm{T} 12$ & Antibiotic & Medical & $\mathrm{N} / \mathrm{A}$ & MRSA & N/A & $\mathrm{N} / \mathrm{A}$ & 8 \\
\hline 11 & $71 / \mathrm{M}$ & $\mathrm{T} 11-\mathrm{T} 12$ & Antibiotic & Medical & $\mathrm{N} / \mathrm{A}$ & MRSA & $\mathrm{N} / \mathrm{A}$ & $\mathrm{N} / \mathrm{A}$ & 8 \\
\hline 12 & $61 / \mathrm{M}$ & L1-L2 & Antibiotic & Medical & N/A & Negative & $\mathrm{N} / \mathrm{A}$ & $\mathrm{N} / \mathrm{A}$ & 6 \\
\hline 13 & $65 / \mathrm{M}$ & $\mathrm{L} 1-\mathrm{L} 2$ & Antibiotic & Medical & $\mathrm{N} / \mathrm{A}$ & Negative & $\mathrm{N} / \mathrm{A}$ & $\mathrm{N} / \mathrm{A}$ & 6 \\
\hline 14 & $44 / \mathrm{M}$ & T12-L1 & Antibiotic & Medical & $\mathrm{N} / \mathrm{A}$ & GPC & $\mathrm{N} / \mathrm{A}$ & $\mathrm{N} / \mathrm{A}$ & 6 \\
\hline 15 & $45 / \mathrm{M}$ & $\mathrm{T} 11-\mathrm{T} 12$ & Antibiotic & Medical & $\mathrm{N} / \mathrm{A}$ & MRSA & $\mathrm{N} / \mathrm{A}$ & $\mathrm{N} / \mathrm{A}$ & 6 \\
\hline 16 & $47 / \mathrm{M}$ & T11-T12 & Antibiotic & Medical & $\mathrm{N} / \mathrm{A}$ & Alpha strep & $\mathrm{N} / \mathrm{A}$ & $\mathrm{N} / \mathrm{A}$ & 6 \\
\hline
\end{tabular}

Abbreviations: Abx, antibiotics; EBL, estimated blood loss; ESBL, extended-spectrum $\beta$-lactamase; GBS, group B Streptococcus; GPC, Gram-positive cocci; LOS, length of stay; MRSA, methicillin-resistant Staphylococcus aureus; MSSA, methicillin-susceptible $S$ aureus; N/A, not applicable; TB, tuberculosis.

ately. A total of 4 patients had diabetes, 1 patient had end-stage renal disease, 2 patients were intravenous drug users, and 2 patients had sacral ulcers. These are all risk factors for the development of spinal infection. In this study, there was no significant correlation between preoperative medical condition and extent of osteomyelitis-discitis or progression of kyphosis. The Table shows baseline clinical variables.

Figure 1 displays the distribution of patients. At initial presentation, surgical intervention, in addition to intravenous antibiotics, was performed in 4 patients. Surgery included one or more levels of decompressive laminectomies only. All 4 patients

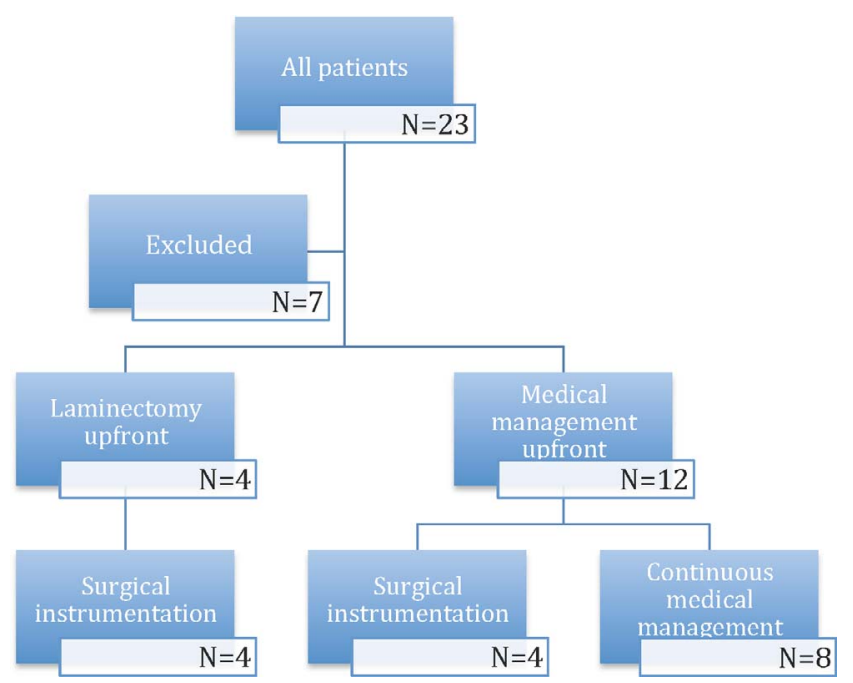

Figure 1. Distribution of patients in the study. later needed surgical instrumentation for kyphosis progression at the level of decompression. The remaining 12 patients were initially treated with antibiotics only. Following initial medical management, 4 of 12 patients needed surgical instrumentation because of the progression of kyphosis at level of disease. Of all the 8 surgical patients, 1 patient had a posterior-only approach (corpectomy/kyphectomy + fusion), whereas 7 patients had a combined anterior-posterior approach (corpectomy + fusion). Figure 2, A and B, shows representative cases of surgical stabilization.

Surgical decompression, epidural compression (Bilsky grades 2 and 3), and vertebral body collapse of greater than $50 \%$ at diagnosis were significant risk factors associated with kyphosis progression requiring surgical instrumentation (Figure 3 ).

The average follow-up duration was 13.2 months, and there was no reoperation due to hardware failure in any of our patients. Patients were followed throughout their duration of hospitalization and seen in clinic after completion of antibiotics and again 6 months (or more) later. For the patients requiring surgical instrumentation due to kyphosis progression, there was a significant difference in the time to kyphosis progression between patients initially treated with antibiotic only versus those treated with laminectomy. The average time to surgical intervention for the indication of progressive kyphosis was 2.6 months after prior laminectomy and 6 months after 

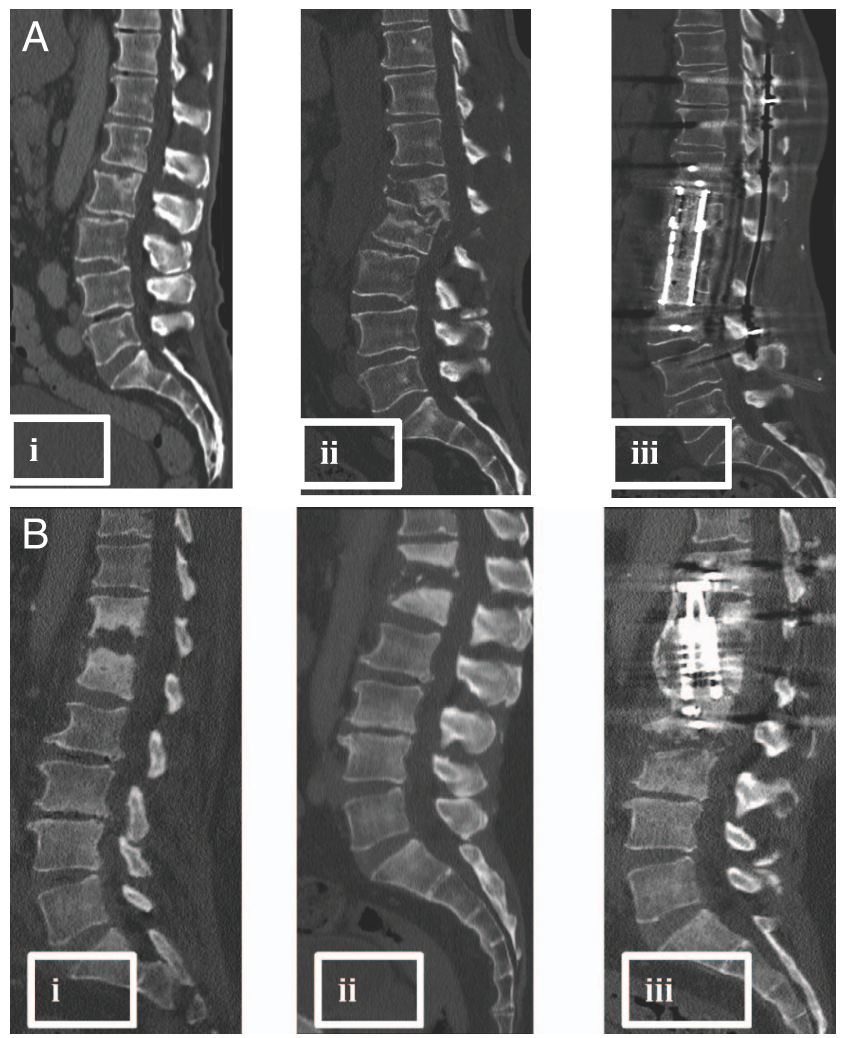

Figure 2. (Ai) Images at initial diagnosis of osteomyelitis-discitis at L1 to L2 with epidural component and compression of neural elements-initial plan for urgent decompression. (Aii) Status after L1 to L2 laminectomy, now with progression of kyphosis. (Aiii) Status after instrumentation and fusion for correction of deformity. (Bi) Images at initial diagnosis of osteomyelitis-discitis at T12 to L1 with minimal epidural component-initial plan to medically manage. (Bii) Status after 8 weeks of antibiotic treatment only, now with progressive kyphotic deformity and neurologic decline. (Biii) Status after instrumentation and fusion for correction of deformity.

initiation of medical management, $P<.001$. This is represented in Figure 4.

There was no statistically significant difference in Cobb angle at diagnosis between patients who later required surgical instrumentation $\left(13.8^{\circ}\right)$ and those only treated medically $\left(13.6^{\circ}\right)$. At follow-up, on average, there was significantly more progression of

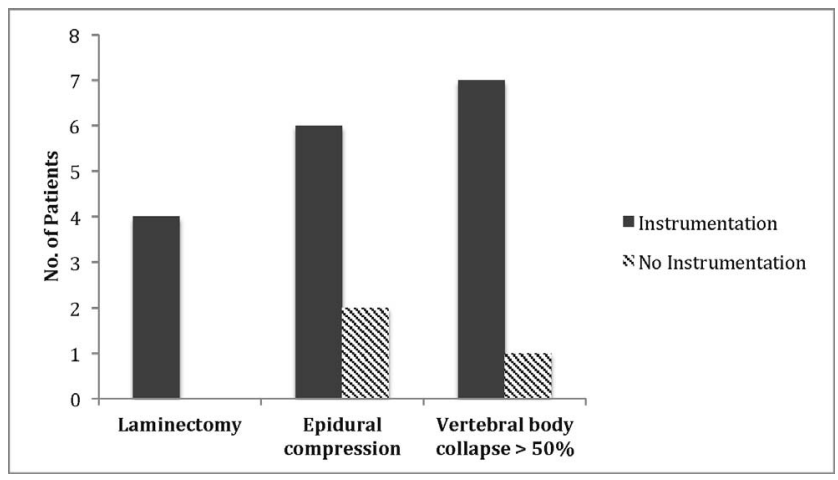

Figure 3. Risk Factors for Instrumentation: Laminectomy, Epidural Compression and Vertebral body collapse

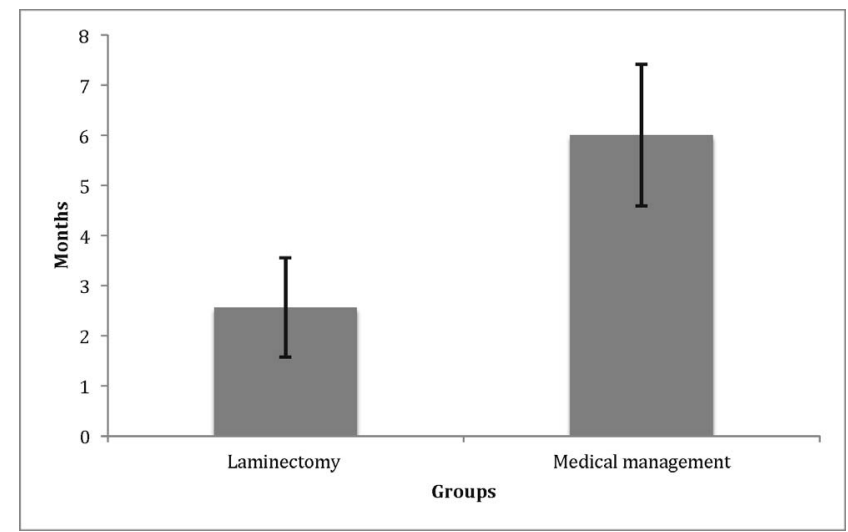

Figure 4. Time to kyphosis progression requiring surgical intervention in patients initially managed medically and with laminectomy

kyphotic deformity in the group requiring instrumentation $\left(46.55^{\circ}\right)$ compared with the medical management-only group $\left(19.17^{\circ}\right), P<0.001$. The average segmental kyphotic deformity improved by $36^{\circ}$ with long construct instrumentation and fusion (Figure 5).

\section{DISCUSSION}

Maintaining neurologic functionality and structural stability of the spinal column remains the primary objective of surgery when there is a destructive pathology of the spine, regardless of the etiology. Long duration of intravenous antibiotics continues to be a crucial part of the treatment paradigm of spinal osteomyelitis-discitis. ${ }^{10,26} \mathrm{Un}$ derstanding the risk factors for spinal instability from an infectious cause continues to be a challenge. In a study by Srinivasan et $\mathrm{al}^{27}$ spinal epidural abscess was the only variable associated with a low likelihood of developing spinal deformity. However,

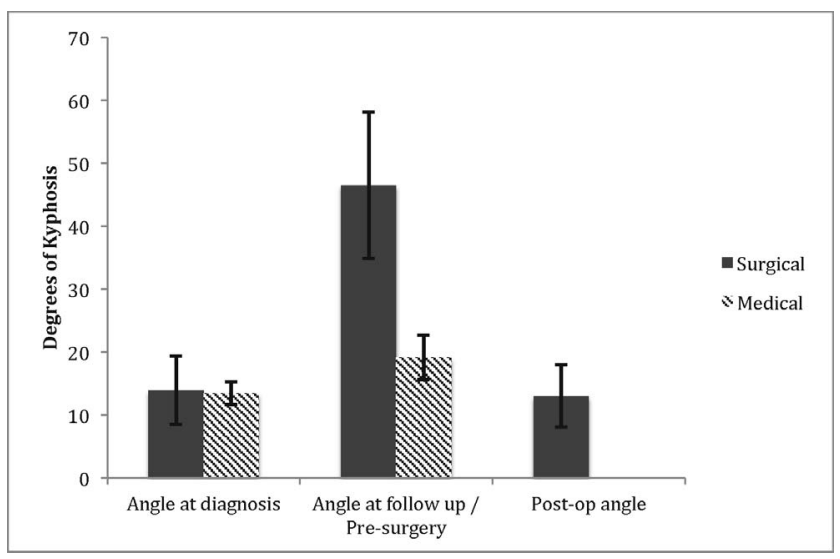

Figure 5. Changes in segmental kyphosis in the medical management only group and in surgical group with resultant improvement in angle of segmental kyphosis after instrumentation 
it is the destructive changes that occur at the intervertebral disc space and vertebral end plates, causing vertebral body collapse, which fundamentally create the higher risk of deformity, thus necessitating immobilization of the affected segment with external orthoses, or internally with surgical fixation. Surgical stabilization has been shown to be superior to external immobilization. ${ }^{18}$ Regarding the appropriate surgical approach, studies have reported good clinical outcomes with an anterioronly approach without posterior fixation, ${ }^{28-31} \mathrm{com}$ bined anterior/posterior approach,,$^{22,28,32}$ anterior approach followed by delayed posterior fixation, ${ }^{22}$ and long posterior fixation. ${ }^{21}$ In the absence of spinal instability at the time of diagnosis, decompression alone could be effective in maintaining neurologic functionality. ${ }^{17}$ At the TL junction, the biomechanical stability relies significantly on the posterior tension band when the vertebral bodies have been destroyed, increasing the baseline risk of deformity if it is not properly managed. There is paucity of data evaluating management of spinal osteomyelitis-discitis at this region and our study evaluates this regional management.

The destructive nature of the underlying infectious process shares some features with traumatic and oncologic injury for which the Thoracolumbar Injury Classification and Severity score ${ }^{33}$ and the Spinal Instability and Neoplastic Score ${ }^{34}$ help facilitate recommendations for management. Similarly to these scoring systems, infectious injury to the spinal column calls for evaluation of vertebral body collapse, integrity of the posterior ligamentous complex, and location of the injury; however, there are many more factors that should contribute to a decision to operate. A similar decision-making approach should be employed in management of spinal osteomyelitis-discitis at the TL junction.

Our study identified 3 factors that increase the risk of kyphotic progression: spinal decompression (laminectomy), epidural compression (grade 2 or 3), and vertebral body collapse $(>50 \%)$. These factors destabilize the spinal column anteriorly and posteriorly, and thus compromise the structural integrity and create a focal zone of weakness where kyphotic deformity endures. Given the increased risk of kyphosis progression, surgical instrumentation should be considered when these risk factors are present.

In our study, the patients who initially presented with epidural compression, with acute neurologic decline, and without focal misalignment underwent emergent decompressive laminectomies without reconstruction. This approach allows for immediate decompression of the spinal cord and nerve roots to aid in neurologic recovery. Patients without neurologic deficits or significant epidural compression were treated with antibiotics and close follow-up. Those who received initial decompressive laminectomy only had faster progression of kyphosis, necessitating instrumentation and fusion for deformity stabilization. This is similar to the poor surgical outcomes reported by Eismont et $\mathrm{al}^{35}$ in patients who had decompression alone. Despite Noh et $\mathrm{al}^{17}$ showing decompression alone to be an effective treatment option in the absence of instability at initial diagnosis, it remains unclear whether such findings pertain to the thoracolumbar junction, because the study did not specify which spinal levels were decompressed. Surgical decompression causes iatrogenic disruption of the posterior tension band, which when coupled with compromised anterior column support from infectious damage, creates progressive instability from kyphotic deformity. Our study suggests that when there are destructive changes in the vertebral end plates and epidural compression despite the absence of overt spinal instability, surgical instrumentation with decompression should be considered for stability preservation and prevention of kyphosis.

Time to progression of kyphotic deformity was another feature analyzed in our study. After initial treatment at diagnosis (decompressive laminectomy or medical management), the average time to progression of kyphosis was 2.6 months and 6 months, respectively. Not only are patients with laminectomy reliably progressing to kyphosis, they do so much faster than patients managed conservatively with intravenous antibiotics only. This again speaks to the dangers of laminectomy alone at the thoracolumbar junction regardless of baseline instability at the time of surgery. The ongoing destructive changes in the spinal column at the infected level create a focus of weakness where the moment arm of any dynamic instability rests in its epicenter. As a result, adequate management entails source control with anterior debridement, which will subsequently require posterior fixation as well.

In our series, instrumented fusion for kyphotic deformity improved overall kyphosis by about $36^{\circ}$, which is significantly more than reported corrections in the literature. ${ }^{15,16,21,32}$ This is in part due to 
the delayed timing of surgical instrumentation in our study. Most of our patients were initially managed conservatively with antibiotics only or antibiotics and decompressive laminectomy. Hence there is a longer time for destructive changes to manifest in the spinal column. Moreover, the presence of laminectomy further compromised spinal stability resulting in worsening kyphotic deformity.

Limitations of this study include its retrospective and nonrandomized design. The sample size was small because of the specific nature of the study in reviewing only cases of vertebral osteomyelitisdiscitis at the thoracolumbar junction as opposed to the entire spine. This makes it difficult to assess the influence of the many factors that ultimately contribute to management. Furthermore, overall systemic response to infection, such as complete blood count, erythrocyte sedimentation rate, and creactive protein, were not consistently documented in all patients. Lastly, pain and functional outcome variables, such as Oswestry Disability Index, visual analog scale, and SF-36, were not adequately and consistently recorded in these patients. Despite these limitations, we believe that our study provides additional clinical insight into the decision-making in management of osteomyelitis-discitis at the thoracolumbar junction. A larger, prospective, multi-institutional study will be needed in the future to corroborate our findings and expand applicability in clinical decision-making. Future directions will also include biomechanical studies to quantify the integrity of the TL junction after laminectomy while simulating vertebral osteomyelitis-discitis. We focused on the TL junction because there is lack of data addressing laminectomy versus instrumentation for vertebral osteomyelitis. We understand this is a small subset of patients undergoing secondary fusion at the TL junction. Our recommendations mostly stand for vertebral osteomyelitis-discitis where the anterior column support is compromised by infection.

\section{CONCLUSION}

The management of osteomyelitis-discitis at the thoracolumbar junction continues to be a therapeutic dilemma. Our study showed that the surgical decompression, epidural compression, and vertebral body collapse are factors that increase the risk of kyphosis progression requiring instrumentation. In addition, laminectomy accelerates kyphosis progres- sion. Therefore, at the time of diagnosis, absence of gross instability or severe kyphosis should not preclude the need for instrumentation at the TL junction. We recommend surgical stabilization along with decompression at the initial surgery in those patients requiring operative management for osteomyelitis-discitis at the thoracolumbar junction, with risk factors for kyphosis progression.

\section{REFERENCES}

1. Carragee EJ. Pyogenic vertebral osteomyelitis. J Bone Joint Surg Am. 1997;79(6):874-880.

2. Jellis JE. Bacterial infections: bone and joint tuberculosis. Baillieres Clin Rheumatol. 1995;9(1):151-159.

3. Rezai AR, Woo HH, Errico TJ, Cooper PR. Contemporary management of spinal osteomyelitis. Neurosurgery. 1999;44(5):1018-1025; discussion 1025-1026.

4. Butler JS, Shelly MJ, Timlin M, Powderly WG, O'Byrne JM. Nontuberculous pyogenic spinal infection in adults: a 12year experience from a tertiary referral center. Spine. 2006;31(23):2695-2700.

5. Acosta FL Jr, Chin CT, Quinones-Hinojosa A, Ames CP, Weinstein PR, Chou D. Diagnosis and management of adult pyogenic osteomyelitis of the cervical spine. Neurosurg Focus. 2004;17(6):E2

6. Gouliouris T, Aliyu SH, Brown NM. Spondylodiscitis: update on diagnosis and management. J Antimicrob Chemother. 2010;65(suppl 3):iii11-24.

7. Pola E, Logroscino CA, Gentiempo M, et al. Medical and surgical treatment of pyogenic spondylodiscitis. Eur Rev Med Pharmacol Sci. 2012;16(suppl 2):35-49.

8. An HS, Seldomridge JA. Spinal infections: diagnostic tests and imaging studies. Clin Orthop Relat Res. 2006;444:27-33.

9. Carragee EJ. The clinical use of magnetic resonance imaging in pyogenic vertebral osteomyelitis. Spine. 1997;22(7):780-785.

10. Berbari EF, Kanj SS, Kowalski TJ, et al. Executive summary: 2015 Infectious Diseases Society of America (IDSA) clinical practice guidelines for the diagnosis and treatment of native vertebral osteomyelitis in adults. Clin Infect Dis. 2015;61(6):859-863.

11. Emery SE, Chan DP, Woodward HR. Treatment of hematogenous pyogenic vertebral osteomyelitis with anterior debridement and primary bone grafting. Spine. 1989;14(3):284291.

12. Liebergall M, Chaimsky G, Lowe J, Robin GC, Floman Y. Pyogenic vertebral osteomyelitis with paralysis: prognosis and treatment. Clin Orthop Relat Res. 1991(269):142-150.

13. Abramovitz JN, Batson RA, Yablon JS. Vertebral osteomyelitis: the surgical management of neurologic complications. Spine. 1986;11(5):418-420.

14. Hsieh PC, Wienecke RJ, O’Shaughnessy BA, Koski TR, Ondra SL. Surgical strategies for vertebral osteomyelitis and epidural abscess. Neurosurg Focus. 2004;17(6):E4.

15. Tsai TT, Yang SC, Niu CC, et al. Early surgery with antibiotics treatment had better clinical outcomes than antibiotics treatment alone in patients with pyogenic spondylodiscitis: 
a retrospective cohort study. BMC Musculoskelet Disord. 2017;18(1):175.

16. Gorensek M, Kosak R, Travnik L, Vengust R. Posterior instrumentation, anterior column reconstruction with single posterior approach for treatment of pyogenic osteomyelitis of thoracic and lumbar spine. Eur Spine J. 2013;22(3):633-641.

17. Noh SH, Zhang HY, Lim HS, Song HJ, Yang KH. Decompression alone versus fusion for pyogenic spondylodiscitis. Spine J. 2017;17(8):1120-1126.

18. Nasto LA, Colangelo D, Mazzotta V, et al. Is posterior percutaneous screw-rod instrumentation a safe and effective alternative approach to TLSO rigid bracing for single-level pyogenic spondylodiscitis? Results of a retrospective cohort analysis. Spine J. 2014;14(7):1139-1146.

19. Korovessis P, Repantis T, Iliopoulos P, Hadjipavlou A. Beneficial influence of titanium mesh cage on infection healing and spinal reconstruction in hematogenous septic spondylitis: a retrospective analysis of surgical outcome of twenty-five consecutive cases and review of literature. Spine. 2008;33(21):E759E767.

20. Madhavan K, Vanni S, Williams SK. Direct lateral retroperitoneal approach for the surgical treatment of lumbar discitis and osteomyelitis. Neurosurg Focus. 2014;37(2):E5.

21. Lin CP, Ma HL, Wang ST, Liu CL, Yu WK, Chang MC. Surgical results of long posterior fixation with short fusion in the treatment of pyogenic spondylodiscitis of the thoracic and lumbar spine: a retrospective study. Spine. 2012;37(25):E1572-E1579.

22. Dimar JR, Carreon LY, Glassman SD, Campbell MJ, Hartman MJ, Johnson JR. Treatment of pyogenic vertebral osteomyelitis with anterior debridement and fusion followed by delayed posterior spinal fusion. Spine. 2004;29(3):326-332; discussion 332.

23. Przybylski GJ, Sharan AD. Single-stage autogenous bone grafting and internal fixation in the surgical management of pyogenic discitis and vertebral osteomyelitis. J Neurosurg. 2001;94(1 suppl):1-7.

24. Kuklo TR, Potter BK, Bell RS, Moquin RR, Rosner MK. Single-stage treatment of pyogenic spinal infection with titanium mesh cages. J Spinal Disord Tech. 2006;19(5):376-382.

25. Ruf M, Stoltze D, Merk HR, Ames M, Harms J. Treatment of vertebral osteomyelitis by radical debridement and stabilization using titanium mesh cages. Spine. 2007;32(9):E275-E280.

26. Johnson JA, Coons SJ. Comparison of the EQ-5D and SF-12 in an adult US sample. Qual Life Res. 1998;7(2):155-166.

27. Srinivasan D, Terman SW, Himedan M, Dugo D, La Marca F, Park P. Risk factors for the development of deformity in patients with spinal infection. Neurosurg Focus. 2014;37(2):E2.

28. Linhardt O, Matussek J, Refior HJ, Krodel A. Longterm results of ventro-dorsal versus ventral instrumentation fusion in the treatment of spondylitis. Int Orthop. 2007;31(1):113-119.

29. Fayazi AH, Ludwig SC, Dabbah M, Bryan Butler R, Gelb DE. Preliminary results of staged anterior debridement and reconstruction using titanium mesh cages in the treatment of thoracolumbar vertebral osteomyelitis. Spine J. 2004;4(4):388395.

30. Krodel A, Kruger A, Lohscheidt K, Pfahler M, Refior HJ. Anterior debridement, fusion, and extrafocal stabilization in the treatment of osteomyelitis of the spine. J Spinal Disord. 1999;12(1):17-26.

31. McHenry MC, Easley KA, Locker GA. Vertebral osteomyelitis: long-term outcome for 253 patients from 7 Cleveland-area hospitals. Clin Infect Dis. 2002;34(10):13421350.

32. Safran O, Rand N, Kaplan L, Sagiv S, Floman Y. Sequential or simultaneous, same-day anterior decompression and posterior stabilization in the management of vertebral osteomyelitis of the lumbar spine. Spine. 1998;23(17):18851890.

33. Vaccaro AR, Lehman RA Jr, Hurlbert RJ, et al. A new classification of thoracolumbar injuries: the importance of injury morphology, the integrity of the posterior ligamentous complex, and neurologic status. Spine. 2005;30(20):2325-2333.

34. Fisher CG, DiPaola CP, Ryken TC, et al. A novel classification system for spinal instability in neoplastic disease: an evidence-based approach and expert consensus from the Spine Oncology Study Group. Spine. 2010;35(22):E1221E1229.

35. Eismont FJ, Bohlman HH, Soni PL, Goldberg VM, Freehafer AA. Pyogenic and fungal vertebral osteomyelitis with paralysis. J Bone Joint Surg. 1983;65(1):19-29.

Disclosures and COI: Dr Ropper is a Consultant for Globus Medical and Stryker. However, this has no bearing on this manuscript. The other authors report no conflicts of interest.

Corresponding Author: Alexander E. Ropper, MD, Baylor College of Medicine, McNair Campus, Department of Neurosurgery, 7200 Cambridge Street, Suite 9A, Houston, TX 77030. Phone: (713) 798-5421. Email: alexander.ropper@bcm.edu.

Published 28 August 2020

This manuscript is generously published free of charge by ISASS, the International Society for the Advancement of Spine Surgery. Copyright (C) 2020 ISASS. To see more or order reprints or permissions, see http://ijssurgery.com. 